\title{
Schistosoma mansoni
} Infection-Induced Transcriptional Changes in Hepatic Macrophage Metabolism Correlate With an Athero-Protective Phenotype

\author{
Diana Cortes-Selva ${ }^{1}$, Andrew F. Elvington ${ }^{2,3}$, Andrew Ready ${ }^{1}$, Bartek Rajwa ${ }^{4}$, \\ Edward J. Pearce ${ }^{5}$, Gwendalyn J. Randolph ${ }^{2}$ and Keke C. Fairfax ${ }^{1,6 *}$
}

${ }^{1}$ Department of Comparative Pathobiology, College of Veterinary Medicine, Purdue University, West Lafayette, IN, United States, ${ }^{2}$ Department of Pathology and Immunology, Washington University School of Medicine, St. Louis, MO, United States, ${ }^{3}$ Division of Health and Sport Sciences, Missouri Baptist University, St. Louis, MO, United States,

${ }^{4}$ Department of Basic Medical Sciences, Bindley Bioscience Center, College of Veterinary Medicine, Purdue University, West Lafayette, IN, United States, ${ }^{5}$ Department of Immunometabolism, Faculty of Biology, Max Planck Institute of Immunobiology and Epigenetics, University of Freiburg, Freiburg, Germany, ${ }^{6}$ Division of Microbiology and Immunology, Department of Pathology, University of Utah School of Medicine, Salt Lake City, UT, United States

\section{OPEN ACCESS}

Edited by:

Thiago Almeida Pereira, Stanford University, United States

Reviewed by: Jose Roberto Machado-Silva, Rio de Janeiro State University, Brazil

Hermelijn Helene Smits,

Leiden University, Netherlands

${ }^{*}$ Correspondence:

Keke C. Fairfax

keke.fairfax@path.utah.edu

Specialty section:

This article was submitted to

Microbial Immunology,

a section of the journal

Frontiers in Immunology

Received: 25 June 2018 Accepted: 19 October 2018 Published: 12 November 2018

Citation:

Cortes-Selva D, Elvington AF,

Ready A, Rajwa B, Pearce EJ,

Randolph GJ and Fairfax KC (2018)

Schistosoma mansoni Infection-Induced Transcriptional

Changes in Hepatic Macrophage Metabolism Correlate With an

Athero-Protective Phenotype.

Front. Immunol. 9:2580. doi: 10.3389/fimmu.2018.02580
Hepatic macrophages play an essential role in the granulomatous response to infection with the parasitic helminth Schistosoma mansoni, but the transcriptional changes that underlie this effect are poorly understood. To explore this, we sorted the two previously recognized hepatic macrophage populations (perivascular and Kupffer cells) from naïve and S. mansoni-infected male mice and performed microarray analysis as part of the Immunological Genome Project. The two hepatic macrophage populations exhibited remarkably different genomic profiles. However, this diversity was substantially reduced following infection with S. mansoni, and in fact, both populations demonstrated increases in transcripts of the monocyte lineage, suggesting that both populations may be replenished by monocytes following infection. Pathway analysis showed a profound alteration in global metabolic pathways, including changes to phospholipid and cholesterol metabolism, as well as amino acid biosynthesis and glucagon signaling. These changes suggest a possible mechanism for the previously reported athero-protective effects of $S$. mansoni infection. Indeed, we find that male ApoE null mice fed a high-fat diet in combination with $S$. mansoni infection have reduced plaque area and increased glucose tolerance as compared to control mice. Transcript analysis of infected and control high-fat diet fed $\mathrm{ApoE}^{-/-}$mice confirm that ApoC1, Psat1, and Gys 1 are all altered by infection, suggesting that altered hepatic macrophage metabolism is associated with S. mansoni- induced protection from hyperlipidemia, atherosclerosis, and glucose intolerance. These results suggest a previously unknown and unreported role of hepatic macrophages in the modulation of whole body lipid and glucose metabolism during infection and provide a template for examining the role of immunomodulation on the long-term metabolism of the host.

Keywords: schistosomiasis, hepatic macrophages, metabolism, helminth, alternative activation of macrophages, atherosclerosis 


\section{INTRODUCTION}

Macrophages are highly plastic cells with vital functions in host defense and tissue repair and homeostasis (1). Their distinct functional characteristics depend on their distribution in different anatomical sites as well as the polarization signals from various tissue milieu (2-4). Due to their heterogeneity, a common classification of "classically activated" and "alternatively activated" has been attributed depending on their characteristic expression markers. While the classically activated macrophages (M1 macrophages) mediate autoimmunity and protection against different bacteria and viruses, alternatively activated macrophages (M2 macrophages) have been shown to play a role in wound healing, the regulation of inflammation, and metabolic functions $(5,6)$. Driven primarily by Toll-like receptor agonists and IFN $\gamma$, M1 polarized macrophages express inducible nitric oxide synthase (iNOS; Nos2) amongst other genes, and are pro-inflammatory, whereas M2 macrophages are induced by IL- 4 and IL-13 and differentially express a range of genes including arginase-1 (Arg1), resistin like beta (Fizz-1; Retnla) and Ym1 (Chil3) (7). M2 macrophages have previously been shown to play a role in both the defense against some parasitic infections, as well as the transition to chronic helminth infection when immunomodulation and tissue repair is crucial for host survival (8). Importantly, they are the predominant macrophage in the liver during the Th2 dominated immune response to the helminth Schistosoma mansoni. In schistosomiasis, M2 macrophages are essential for survival of acute infection $(9,10)$ and provide proline, a collagen precursor, while also aiding in both the development and modulation of fibrosis and liver pathology (7). Recent studies have established that Arginase1 producing M2 macrophages are essential for suppressing Th2 driven inflammation and fibrosis (11), and our recent work identified immune complex-driven production of IL-10 by these macrophages as critical for immunomodulation in chronic infection (12). However, knowledge about the transcriptional profile of distinct macrophage subsets during schistosome infection remains limited.

Mice infected with $S$. mansoni serve as a reproducible model for human schistosomiasis. In both humans and mice, the pathology progresses from an early Th1 response into a Th2 dominated one in response to egg deposition in organs such as liver, intestines, and lungs by worm pairs. Eggs are highly immunogenic and toxic, and the host response to this stimulus leads to fibrosis and portal hypertension (13). Previous studies have reported that schistosome infection in both humans and mice correlates with a significant modulation of lipid metabolism (14), lowering the total cholesterol levels of randomly bred mice and ApoE deficient mice (15), and reducing atherosclerotic lesions in ApoE deficient mice (16). Despite previous observations of the correlation of schistosome infection and lipid metabolic changes in both human and murine models, the mechanism by which $S$. mansoni exerts protection against cardiovascular disease is not understood.

Here we describe microarray data of the transcriptional profiles of hepatic macrophages in naïve and S. mansoni infected mice that indicate a reduction in the transcriptional heterogeneity of both Kupffer cells and perivascular macrophages following schistosome infection that suggests a shared function for these distinct macrophage populations. Surprisingly, a large percentage of the transcriptional changes observed in macrophages relate to genes involved in cholesterol and phospholipid metabolism, including ApoC1. These data suggest a pivotal role of hepatic macrophages in the modulation of lipid metabolism during infection. Furthermore, we confirm the antiatherogenic effect of active schistosome infection in an $\mathrm{ApoE}^{-/-}$ atherosclerosis mouse model and postulate that this protection is due in part to significant reductions in serum ApoC1. Finally, we demonstrate that $S$. mansoni infection leads to a notable reduction in high fat diet (HFD) induced insulin resistance, and identify transcriptional changes to amino acid and glycogen metabolism that are likely mechanisms for this protection. Altogether, the data suggest an important and unexplored role of liver macrophages in the regulation of atherosclerosis and insulin sensitivity, a finding that has significant implications for diabetes, obesity and many other human diseases involving hyperlipidemia and insulin resistance.

\section{MATERIALS AND METHODS}

\section{Ethics Statement}

This study was carried out in strict accordance with the recommendations in the Guide for the Care and Use of Laboratory Animals of the National Institutes of Health. The protocols were approved by the Institutional Animal Care and Use Committees of Washington University in St. Louis and Purdue University.

\section{Mice And Parasites}

Male C57BL/6J mice were purchased from Jackson Laboratories, ApoE $E^{-/-}$(B6.129P2-Apoetm1Unc/J) mice were bred at Purdue University. Mice were kept under specific pathogen-free conditions and male mice were infected at 6 weeks of age. Snails infected with S. mansoni (strain NMRI, NR-21962) were provided by the Schistosome Research Reagent Resource Center for distribution by BEI Resources, NIAID NIH. Mice were exposed percutaneously to $75 \mathrm{~S}$. mansoni cercariae. For experiments using $A \mathrm{poe}^{-/-}$mice, animals were transitioned to HFD (21\% milk fat, $0.15 \%$ cholesterol; TD 88137 Harlan Teklad) 10 days prior to $S$. mansoni infection. Control mice were fed standard rodent chow (2018 rodent chow, Harlan Teklad) and mice were sacrificed at 10 -weeks post-infection.

\section{Macrophage Identification and Isolation}

Livers were removed from PBS-perfused animals, mashed, and incubated in RPMI (Mediatech) containing $250 \mu \mathrm{g} / \mathrm{ml}$ Collagenase D (Roche) at $37^{\circ} \mathrm{C}$ for $60 \mathrm{~min}$. The resulting suspension was disrupted through a $100 \mu \mathrm{m}$ metal cell strainer, washed, and then red blood cell lysed with ACK lysis buffer (BD) two times to remove hepatocytes followed by washing. The resulting pellet was washed and used for sorting. Surface staining with monoclonal antibodies, acquisition, and sorting were performed according to Immgen standard protocols (www. immgen.org). The following mAb (eBioscience, BioLegend, or 
$\mathrm{R} \& \mathrm{D}$ systems) against mouse antigens were used as $\mathrm{PE}, \mathrm{PE}-\mathrm{Cy} 5$, PE-Cy7, allophycocyanin (APC), APC-Cy7, Pacific blue, or biotin conjugates: CD11c (N418), CD11b (M1/70), CD45 (30-F11), MHC-II (M5/114.15.2), F4/80 (BM8), and MERTK (BAF591). Cells were directly sorted from mouse tissues and were processed from tissue procurement to a second round of sorting into TRizol within $4 \mathrm{~h}$ using a Beckton-Dickinson Aria II instrument.

\section{Microarray Analysis, Normalization, and Dataset Analysis}

RNA was amplified and hybridized on the Affymetrix Mouse Gene 1.0 ST array by the Immgen consortium using doublesorted cell populations sorted directly into TRIzol. Data analysis utilized GenePattern analysis software. Raw data were normalized using the robust multi-array algorithm, returning linear values between 10 and 20,000. A common threshold for positive expression at 95\% confidence across the dataset was determined to be 120. Differential gene expression signatures were identified and visualized using the "Multiplot" module of GenePattern (http://www.broadinstitute. org/cancer/software/genepattern/). Probesets were considered as differentially expressed with a coefficient of variation $<0.5$ and a $p<0.05$ (Student's $T$-test). Calculation and visualization of differential gene expression sizes (calculated as $\log _{2}$-transformed Hedges' g measures accompanied by 95\% confidence intervals and $t$-test $p$-values indicating significance of differences, raw foldchanges, and $\log _{2}$-foldchanges) were performed using $\mathrm{R}$ language for statistical computing.

To identify important genes (i.e., the genes expressed in a matter suggesting a link to macrophage class and/or infection status) we used various Boolean selection strategies focusing on multiple scenarios:

1. Genes which are upregulated in $\mathrm{F} 4 / 80^{\text {high }}$ (Kupffer cells) (foldchange $>0$ ), but downregulated in F4/80 int (perivascular macrophages) (foldchange $<0$ ).

2. Genes which are downregulated in $\mathrm{F} 4 / 80^{\text {high }}$ (foldchange $<0$ ), but upregulated in F4/80 int (foldchange $>0$ )

3. Genes which are significantly (Benjamini-Hochberg adjusted $\mathrm{p}<0.05)$ upregulated in both cell types

4. Genes which are significantly (Benjamini-Hochberg adjusted $\mathrm{p}<0.05$ ) downregulated in both cell type

5. Genes which differ substantially in the degree of regulation (regardless of the direction of regulation).

The described Boolean strategies are illustrated in the Venn diagrams in Figures 2A-C. The following set designations were used in the set notation:

- $\mathrm{q}_{-}$hi $=$Significantly regulated genes in $\mathrm{F} 4 / 80^{\text {high }}$ cells $(\mathrm{BH}$ adjusted $p<0.05$ )

- q_nt $=$ Significantly regulated genes in F4/80 int cells $(\mathrm{BH}$ adjusted $p<0.05$ )

- hi_up $=$ Genes upregulated in F4/80high cells

- int_up $=$ Genes upregulated genes in F4/80 int cells

- hi_down $=$ Genes downregulated in F4/80 high cells

- int_down $=$ Genes downregulated in F4/80 int cells
The employed set notation:

- $\mathrm{A} \cap \mathrm{B}-\mathrm{A}$ intersect $\mathrm{B}$

- $A \cup B-A$ union $B$

- $\mathrm{A} \backslash \mathrm{B}-\mathrm{A}$ minus $\mathrm{B}$, or $\mathrm{A}$ complement $\mathrm{B}$

The Data (significantly impacted pathways, biological processes, molecular interactions.) were analyzed using Advaita Bio's iPathwayGuide (http://www.advaitabio.com/ipathwayguide). Pathway analysis was performed on $\log _{2}$-transformed data using Bonferroni-corrected $p$-values. Data are deposited in GenBank under accession no. GSE37448 as part of the Immgen 2 dataset.

\section{Principle Component Analysis}

For unsupervised visualization of the gene expression profiles via principal component analysis, the gene expression data were constrained to a simplex and transformed using centered log-ratio transformation. The resultant values were ordered according to their variances, and the top 0.5-percentile of the genes were selected for the computation of the principal components (PCs).

\section{Flow Cytometric Analysis}

Livers were perfused with $1 \mathrm{X}$ PBS, mashed and digested in DMEM containing $250 \mu \mathrm{g} / \mathrm{ml}$ Collagenase (Sigma) at $37^{\circ} \mathrm{C}$ for $30 \mathrm{~min}$. The digested livers were then mashed and filtered through a $100 \mu \mathrm{m}$ metal strainer and digestion was repeated for $15 \mathrm{~min}$. Total liver contents were strained and washed with DMEM. The pellet was lysed with $1 \mathrm{X}$ lysis buffer (BD PharmLyse), quenched, and washed. The resulting cell suspension was used in flow cytometry. Surface staining was performed using the following $\mathrm{mAb}$ against mouse antigens: CD45 (30-F11, eBioscience), CD301(BioRad), CD206 (C068C2, Biolegend), F4/80 (BM8, Biolegend), mouse Mer biotinylated (R\&D), CD64(X54-5/7.1, BD). Samples were acquired using FACSCanto II flow cytometer (BD) and analyzed using Flowjo X 10.0.7r2 (FlowJo LLC, Inc.,).

\section{Analysis of Atherosclerotic Plaques}

At sacrifice, mice were perfused with PBS, and the heart was extracted for plaque assessment at the aortic sinus. Following fixation in $4 \%$ paraformaldehyde, tissue was cryoprotected in $30 \%$ sucrose, embedded in OCT compound (Fisher Scientific), and flash frozen. Fifteen-micron cryosections were made in the aortic sinus from initiation to termination of the aortic valve leaflets. Plaque assessment in the aortic sinus was performed as previously described (17), and is briefly described as follows. The total plaque was determined by taking the average of total atherosclerotic plaque from at least five sections at least 60 microns apart. Macrophage content was determined using CD68 immunofluorescence: average CD68 (BioRad) positive area was assessed from at least five sections at least 60 microns apart. Quantification of plaque area and area occupied by CD68 was calculated with ImageJ software.

\section{Cholesterol Quantitation}

Total cholesterol was measured from plasma as previously described (17) using colorimetric assessment methods. In brief, 
whole blood was collected by mandibular bleed into EDTAplasma collection tubes, and the plasma fraction was removed following red blood cell sedimentation via centrifugation. Plasma samples were stored at $-80^{\circ} \mathrm{C}$ until use. Total plasma cholesterol was assessed by manufacturer's protocol (Wako Diagnostics, \#999-02601). Values were calculated from the mean of two replicates from a standard calibration curve. Total plasma cholesterol was reported as mg per $\mathrm{dL}$.

\section{RNA Isolation and q-RT-PCR Analysis}

Sections of perfused livers were homogenized in Trizol, and RNA isolation was performed as previously described [Immunological Genome Project, Total RNA isolation with Trizol; (18)]. RNA was used for cDNA synthesis using Superscript II (Invitrogen) for $\mathrm{qPCR}$ analysis. $\mathrm{qPCR}$ was performed using TaqMan Gene expression assays (gys1, CD36, ApoC1, ThermoFisher) or SYBR Green [psat1 (19), primers forward: ACG CCA AAG GAG ACG AAG CT and reverse: ATG TTG AGT TCT ACC GCC TTG TC] on an Applied Biosystems Stepone Plus Real-Time PCR System. Beta-Actin assay number Mm00607939_s1, GYS1 assay Mm01962575_s1, CD36 assay Mm00432403_m1, ApoC1 assay Mm00431816_m1. Relative expression was calculated using the 2- $\Delta \Delta \mathrm{Ct}$ method.

\section{ELISA}

ApoC1 plasma concentrations were determined by ELISA using an anti-mouse ApoC1 ELISA kit as per the manufacturer's instructions (LSBio).

\section{Statistics}

Statistical analyses for non-microarray data were performed using one-way ANOVA, a non-parametric Mann-Whitney test, or unpaired Student's t-test depending on the distribution of the data. $P \leq 0.05$ were considered statistically significant. Graph generation and statistical analyses were performed using Prism (GraphPad v7.0) and R-language for statistical computing.

\section{RESULTS}

\section{S. mansoni Infection Profoundly Alters Hepatic Macrophage Metabolism}

While it is widely accepted that hepatic macrophages play a critical role in the pathology and ultimately the survival of $S$. mansoni infection, the transcriptional changes that accompany the response to $S$. mansoni antigens are not entirely understood. We have previously established 10-weeks post-infection as a key time-point in immunomodulation where SEA (schistosome egg antigen) specific B cells are recruited to the liver, and immune-complex ligation of hepatic macrophages begins, leading to macrophage IL-10 production (12). To explore the transcriptional changes to liver macrophages associated with $S$. mansoni infection, we first identified hepatic macrophages $\left(\mathrm{CD} 45^{+} \mathrm{PI}^{-} \mathrm{Mertk}^{+} \mathrm{CD}^{+} 4^{+}\right)$and then sorted $\mathrm{F} 4 / 80$ high (Kupffer cells) and F4/80 int (perivascular macrophages) populations from the livers of naïve and 10 -week old $S$. mansoni-infected C57BL/6 mice according to Immgen standard protocols $[(4,20)$, Figures 1A,B]. Cytospin examination of cells from both naïve and infected mice confirmed that both populations were macrophages, with the morphology of F4/80 high macrophages displaying greater size heterogeneity than the F4/80 int macrophages (Figure 1C).

We compared the gene expression profile of naïve Kupffer cells and perivascular macrophages and found very high transcriptional diversity between these two macrophage populations considering that they reside in the same tissue environment (Figure 1D). Subsequently, we performed the same comparison for 10-week post-infection samples and observed that transcript diversity decreases following $S$. mansoni infection (Figure 1E), suggesting that Kupffer cells and perivascular macrophages may have shared functions in response to infection. Additionally, some of the transcripts that increased in both populations following infection (i.e., Ly6C) were consistent with both macrophage populations being repopulated by monocytes at this time-point, as recently described $(21,22)$. A recent report (23) indicated that in models of sterile liver injury, peritoneal macrophages are recruited to the liver to aid in tissue repair, we did not, however, detect increases in Gata6 transcripts in our microarrays, so this paradigm may not apply to $S$. mansoni infection. We then performed an unsupervised visualization of the gene expression profiles before and after $S$. mansoni infection via principal component analysis (Figure 1F). The plot demonstrated that the PC 1 is associated with the difference between cells from the naïve and the 10 -week $S$. mansoni-infected animals, whereas the PC 2 described the difference between the F4/80 high and the F4/80 int cells regardless of the infection status. The ten genes with the highest contribution to PC 1 are Slc7a2, Timd4, Flrt2, F7, Atp6v0d2, Pdcd1lg2, Arg1, Ch25h, ApoC1, and Dhrs9. The 10 genes with the highest contribution to PC 2 are Lum, Lox, Dpt, Cd163, Slc7a11, Igk-V1, Mgp, Jam2, Emcn, and Arhgap29.The effect sizes of the differences in the gene expressions represented as two-dimensional Mahalanobis distances in PC space are 18.83 (naïve F4/80 int cells vs. 10 -week S. mansoni F4/80 int cells), 35.32 (naïve F4/80 high cells vs. 10 -week S. mansoni F4/80high cells). In both cases, the naïve vs. infected differences were statistically significant (Hoteling $\mathrm{T}^{2}$ test, $p<$ $10^{-5}$ ). Although distances for naïve $\mathrm{F} 4 / 80^{\text {high }}$ vs. naïve $\mathrm{F} 4 / 80^{\text {int }}$ cells and 10 -week infected F4/80 high vs. 10-week-infected F4/80 low cells were similar (4.40 and 4.09 , respectively), the larger variance among naïve $\mathrm{F} 4 / 80^{\mathrm{int}}$ cells made the former pair of expressions indistinguishable in the PC space (Hoteling $\mathrm{T}^{2}$ test, $\mathrm{p}>0.1$ ). On the other hand, the dissimilarity between the gene expressions identified in the cells from the infected animals was significant $(p=0.004)$.

Next, we employed the Boolean selection strategies detailed in the methods section to compare the transcriptional profiles of Kupffer cells and perivascular macrophages from 10-week infected animals to their naïve counterparts, and identified numerous subsets of differentially regulated genes (Figure 2). Subsets 3, 4, and 6 shed light on the putative functional roles of these two cell types in response to $S$. mansoni infection. Among

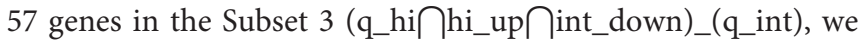
located Cbr2 (carbonyl reductase 2, involved in arachidonic acid metabolism), which is significantly $(p<0.05)$ and highly (fold size $=20.7$ ) upregulated in F4/80 high, but does not demonstrate a statistically significant change in F4/80 int, Alox $(p<0.05$, fold size $=5.09)$, and F13a1 $(p<0.05$, 
A

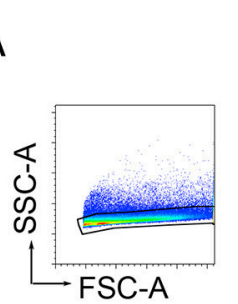

B

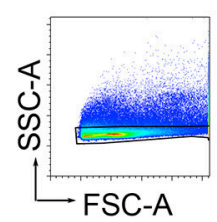

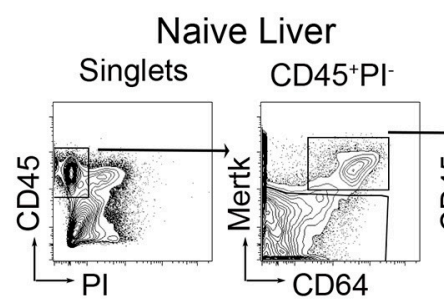

Naive Liver

10 Week S. mansoni Liver

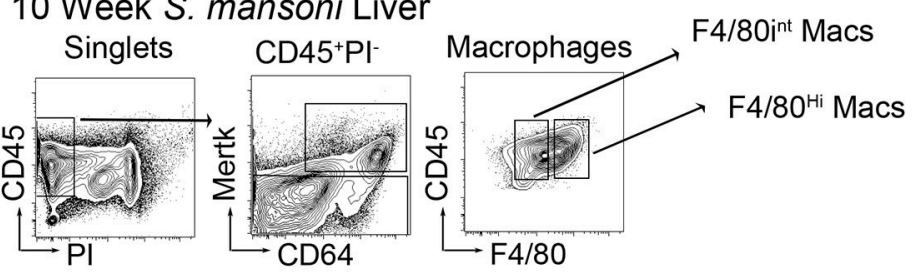

Naive

C $\mathrm{F} 4 / 80^{\mathrm{Hi}}$ Macs

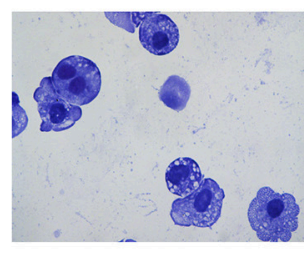

D

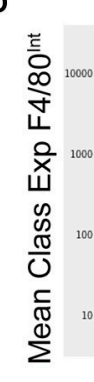

E
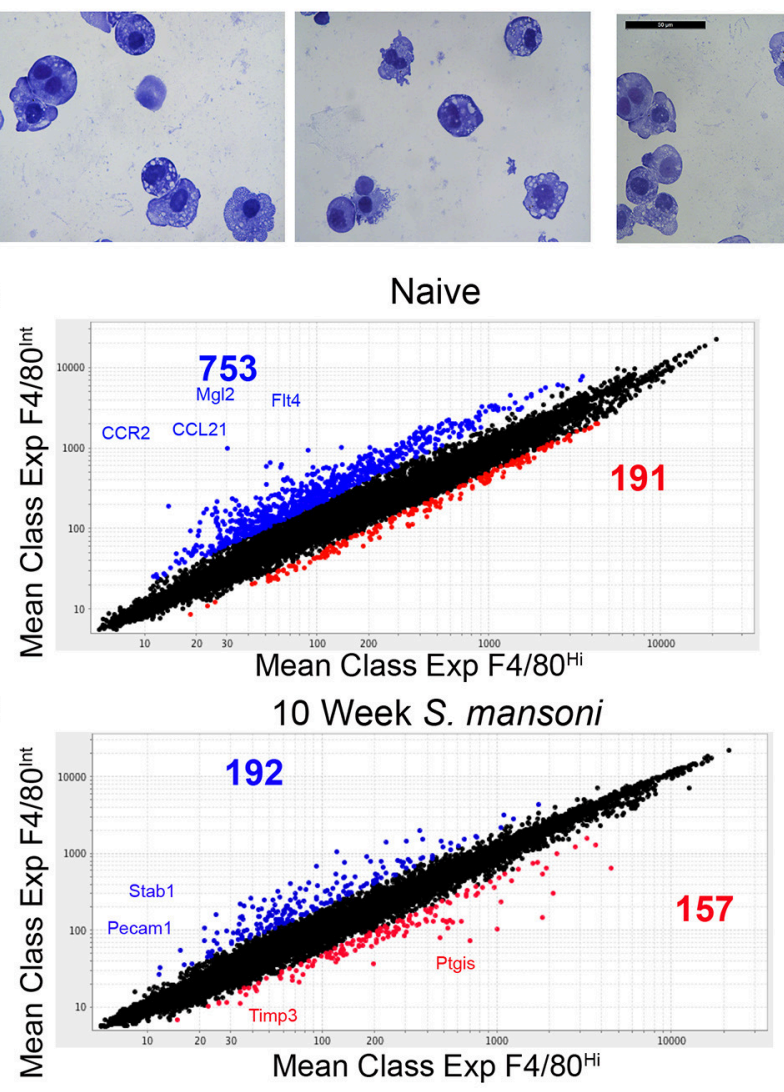

Naive

191
10 Week S. mansoni

F4/80 $0^{\text {Hi Macs }}$

F4/80 ${ }^{\text {Int }}$ Macs
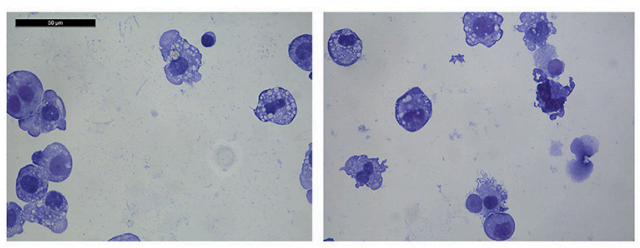

F

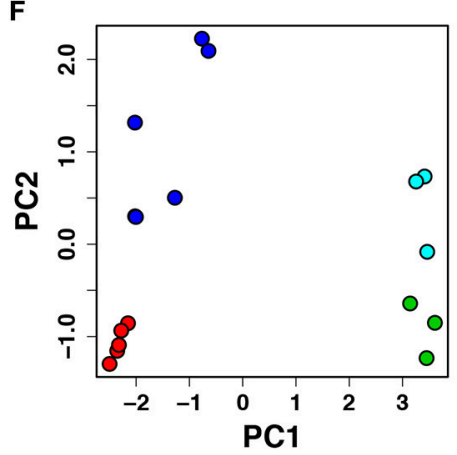

FIGURE 1 | Murine livers contain two distinct macrophage populations and S. mansoni infection decrease transcript diversity between Kupffer cells and perivascular macrophages. C57BL/6 male mice were either naïve or infected with S. mansoni for the indicated number of weeks. (A,B) Perfused livers were removed, digested with collagenase for $1 \mathrm{~h}$, and disrupted through a metal strainer to create single cell suspensions. Cells were identified by staining for the indicated markers and sorted on an Aria II. All populations were sorted two times, with the second sort going directly into Trizol according to the Immgen protocol. Individual animals were sorted from multiple litters/infections to achieve 4-5 biological replicates for individual microarray analysis. (C) Representative cytospins of aliquots from the second sorts of each macrophage populations were stained with H\&E. (D,E) Total RNA was extracted, amplified, and transcript expression determined using whole-mouse genome Affymetrix Mouse Gene 2.0 ST Arrays through the Immunological genome consortium. (D) The number of probes increased by 2-fold with a $p>0.05$ Blue indicates increased expression in F4/80 int liver macrophages at steady state, Red indicates increased expression in F4/80hi liver macrophages at steady state. (E) The number of probes increased by 2 -fold with a $p>0.05$ Blue indicates increased expression in $\mathrm{F} 4 / 80^{\text {int }}$ liver macrophages at steady state, Red indicates increased expression in $\mathrm{F} 4 / 80^{\text {hi }}$ liver macrophages at 10-weeks post $S$. mansoni infection. Each group contained 3-5 individual RNA samples. (F) Principal component plot showing differences in gene expression profiles between samples of naïve F4/80high cells (green), 10-week S. mansoni F4/80high cells (red), naiive F4/80int cells (cyan), and 10-week S. mansoni F4/80int cells (blue). 


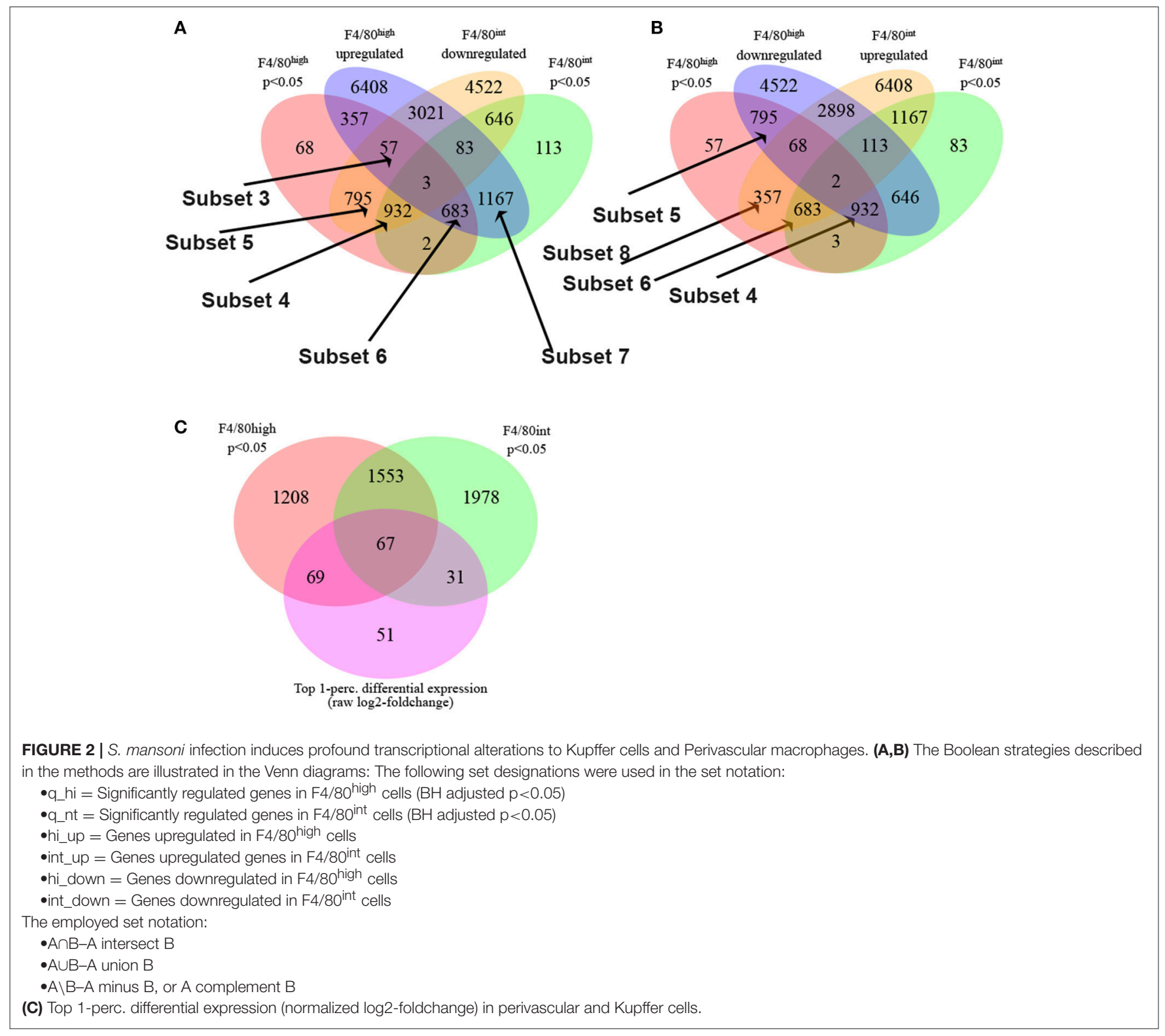

fold size 20). Additionally, among 932 genes in Subset

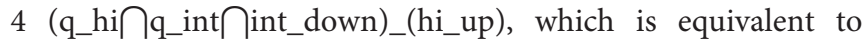

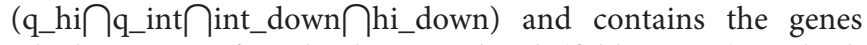
which are significantly downregulated (foldsize $<0$ ) in both cell types we found Adh1, Alb, Aldh2, Apoa2, ApoB, ApoC1, ApoC3, Cd55, Clec4f, Creg1, Fabp7, Flt4, Gpr116, Hmcn1, Hpgd, Marco, Pde7b, Ptprb, Slc16a9, Slc40a1, Slco2a1, St3gal5, and Stab2. Surprisingly, this subset contains many genes involved in phospholipid and cholesterol metabolism, as well as solute transport. Further, we found that Prg4 and Spic belong to a Subset 5 of 795 genes that are downregulated in both macrophage types, but the downregulation in $\mathrm{F} 4 / 80^{\mathrm{int}}$ has not been statistically significant. The set is designated as (q_hi门int_down)_(q_int Uhi_up). 683 genes were significantly simultaneously upregulated in $\mathrm{F} 4 / 80^{\mathrm{int}}$ and $\mathrm{F} 4 / 80^{\mathrm{high}}$ forming

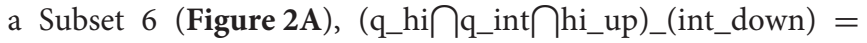

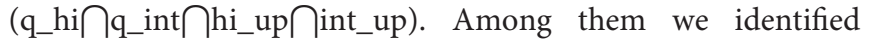
Anxa1, Arg,1 Atp1a3, Ccr2, Cd63, Chi313, Chi314, Dhrs9, Ear11, Egr2, Fcrlb, Fn1, Gda, Mgl2, Nfil3, Nos2, Pdcd1lg2, Retnla, Slc41a2, Slc7a2, and Uck2. This set contains many of the genes that are known to be hallmarks of alternative activation. Our filtering strategy identified 1167 genes belonging to (q_int $\bigcap$ hi_up)_(q_hiUint_down), denoted as Subset 7. These genes demonstrate upregulation in both populations of macrophages; however, the upregulation in $\mathrm{F} 4 / 80^{\text {high }}$ is not statistically significant. Among them, we found Ccl2, $\mathrm{Ccl} 7$, and Ptgs2. Another interesting subset of 357 genes

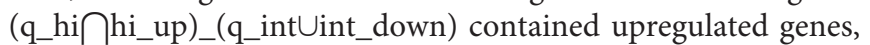
which changed in a statistically significant manner in $\mathrm{F} 4 / 80^{\text {high }}$ but not in $\mathrm{F} 4 / 80^{\text {int }}$. Ccl12 with a foldchange of $\sim 15$ and Ccl8 with 
a very high foldchange of 76.6 in F4/80 ${ }^{\text {high }}$ as well as Mfsd6 were among these genes. Interestingly, there were only three genes in

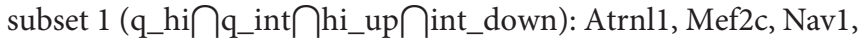

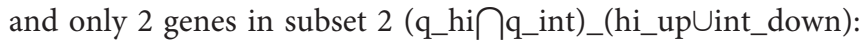
Igf2bp2 and Ube2j2 (Figure 2A), none of these genes had a particularly high absolute fold-change, further suggesting that both perivascular macrophages and Kupffer cells have a shared response to $S$. mansoni infection.

Some genes which were upregulated in both types of macrophages (or downregulated in both) nevertheless showed substantial differences in the level of regulation, exhibited by a large disparity in foldchanges. For instance, Rnase 4 was upregulated in F4/80 high and F4/80 int; however, in the first case the observed foldchange was 11, and in the second instance is only 1.6. To further delineate key differential transcriptional changes, we used the absolute difference between $\log _{2}$-foldchanges as a direct metric of differential regulation. The top 1-percentile of genes sorted by this measure contains 67 transcripts (Figure 2C) expressed with $\log _{2}$-foldchange difference ranging from 2.75 to 1.4. Among them, there are some of the known hallmarks of alternative activation (in the order of differences) Mgl2 (F4/80 ${ }^{\text {high }}$ foldchange $=35.8, \mathrm{~F} 4 / 80^{\text {int }}$ foldchange $=7.5)$, Retnla $\left(\mathrm{F} 4 / 80^{\text {high }}\right.$ foldchange $=84.2, \mathrm{~F} 4 / 80^{\mathrm{int}}$ foldchange $=19.5$ ) as well as Ear11, Chi313, Ptprb, Ccr2, Gda, Gpr116, St3gal5, Stab2, Cd63.

These data prompted us to conduct pathway analysis using the Advaita iPathwayGuide to uncover the pathways that are significantly manipulated by $S$. mansoni infection. Meta-analysis of transcriptional changes shared across both hepatic macrophage populations revealed striking alterations to multiple KEGG pathways, including multiple pathways of inflammation and cellular metabolism (Figure 3A), with the KEGG metabolic pathway showing the highest number of altered genes (Figures 3A,B, pathway Bonferroni corrected $p=1.042 \times 10^{-8}$ and Supplementary Figure 1). While some of these metabolic genes have previously been linked to $S$. mansoni infection (i.e., Arg1 and Nos2), others were not previously considered to be associated with either alternative activation, or S. mansoni infection (i.e., Gys1, Psat1). We conclude that $S$. mansoni infection strongly alters the metabolic potential of liver macrophages. Since the liver is responsible for regulating many aspects of whole body metabolism (24, $25)$, these alterations may have profound effects on infected individuals.

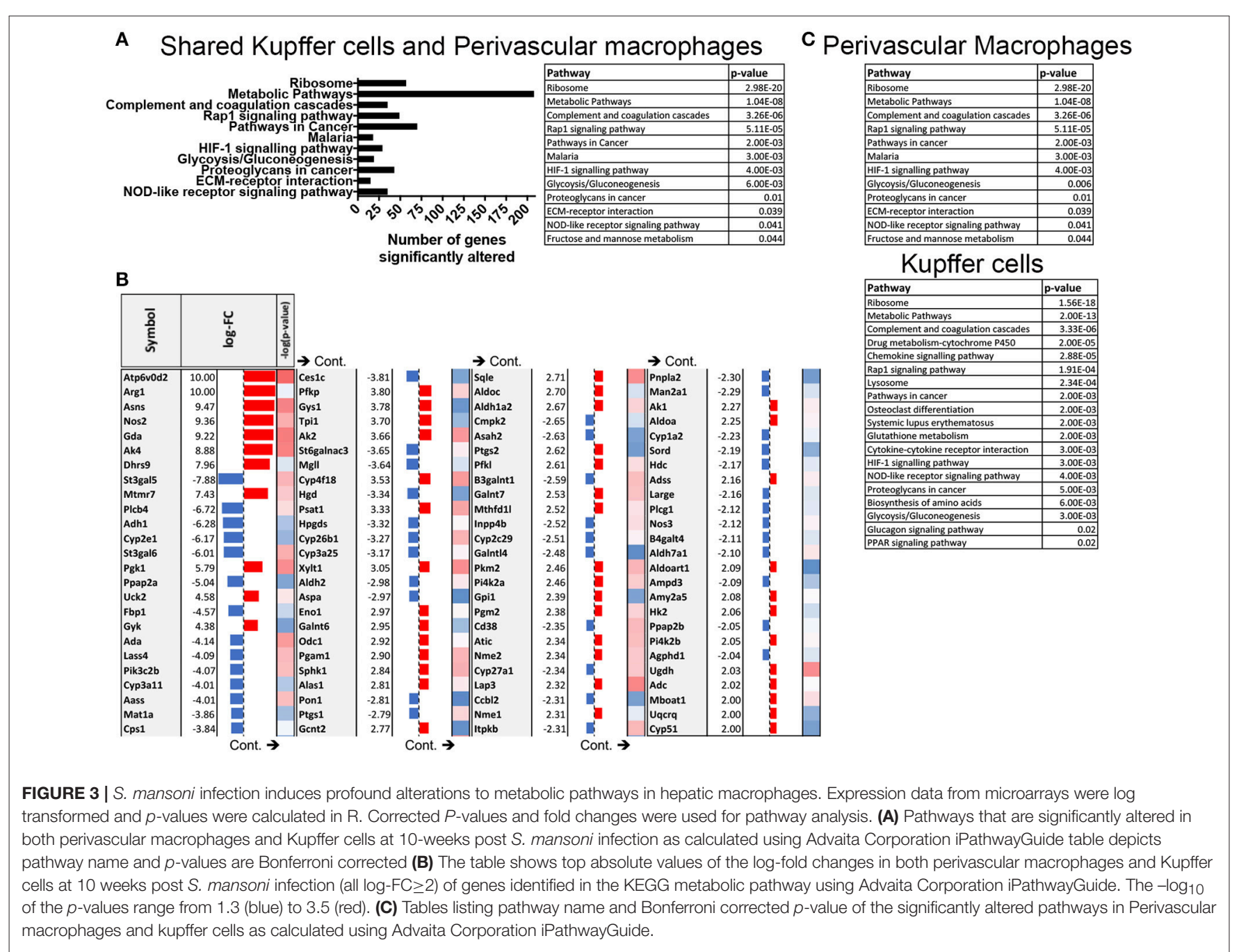




\section{Infection Reduces ApoC1 Production and Aortic Plaque Formation}

Schistosome infection in humans (26) and mice (15, 16, 27) protects from the development of obesity and atherosclerosis, but the molecular mechanism(s) that underlies this protection has yet to be fully uncovered. The transcriptional profile revealed by our analysis suggested that $S$. mansoni-induced metabolic alterations to hepatic macrophages could be involved in infection-induced protection from metabolic diseases. To assess this hypothesis, we employed the $\mathrm{ApoE}^{-/-}$model of hyperlipidemia/atherosclerosis (28). ApoE ${ }^{-/-}$mice were fed either a high-fat Western diet (HFD) or normal chow diet for 10 days before infection with S. mansoni, whereas controls were sham-infected. At 10-weeks post-infection we analyzed CD301 (galactose type C lectin ) and CD206 (mannose receptor) expression as markers of alternative activation. Approximately $40-60 \%$ of macrophages from control chow uninfected were double positive, with the rest being CD206 ${ }^{+}$(Figure 4). Infection increased the frequency of alternative activation to $\sim 80 \%$ regardless of diet, suggesting that HFD do not interfere with $S$. mansoni induced macrophage polarization. Similar to previous reports $(15,16)$, S. mansoniinfected mice on an HFD diet had significantly lower levels of plasma cholesterol and smaller aortic sinus plaques than uninfected HFD mice (Figures 5A,B). Plaques in the aortic sinus of $\mathrm{HFD}$ infected mice also contained fewer $\mathrm{CD} 68^{+}$macrophages (Figure 5C), suggesting reduced recruitment of monocytes to the plaque, as these macrophages have been previously shown to be monocyte-derived $(29,30)$. The transcriptional profile we uncovered in our analysis of schistosome-infected C57BL/6 liver macrophages (Figures 2, 3) showed reduced production of multiple apolipoproteins in macrophages, including ApoC1 $\left(-10.00 \log\right.$-FC, $\left.p=1.35 \times 10^{-5}\right)$ and ApoC3 $(-5.78 \log -$ FC, $p=0.006)$. To confirm gene modulation on the ApoE $\mathrm{E}^{-/-}$ model, we assessed the expression of a list of genes of interest. Importantly, ApoC1 was significantly downregulated in the same manner in both the $\mathrm{C} 57 \mathrm{BL} / 6$ and $\mathrm{ApoE}^{-/-}$model. Additionally, a previous report has indicated that $\mathrm{ApoC} 1$ production drives hyperlipidemia and pathogenesis in the $\mathrm{ApoE}^{-/-}$model (31), making it a likely candidate for correlation with reduced plaque formation during infection. To address this possibility, we examined liver transcripts and plasma levels of ApoC1 and found that HFD induces an increase in ApoC1, but this increase is significantly attenuated by $S$. mansoni infection (Figures 5D,E). This reduction, in systemic ApoC1 production, is a component of the postulated mechanism for $S$. mansoni-related protection from HFD- induced atherosclerosis.

\section{Patent S. mansoni Infection Leads to Improved Glucose Tolerance and Alterations in Hepatic Macrophage Amino Acid Biosynthesis and Glucagon Signaling Pathways}

Schistosomiasis has been reported to correlate with lower hemoglobin A1c levels in populations with a previous history of infection (26), as well in a murine model of obesity (27), so we sought to examine glucose tolerance in this model. $S$. mansoni

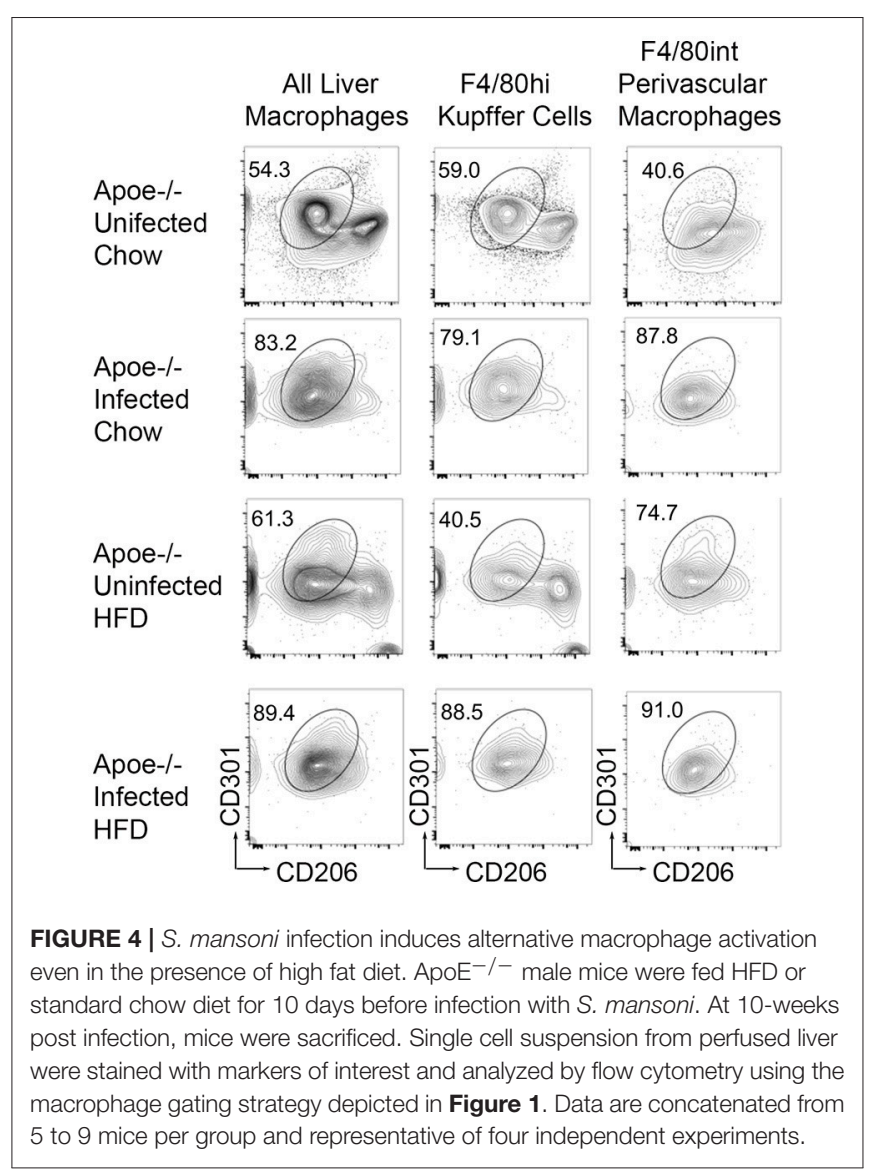

induced reductions in serum cholesterol have previously been shown to be egg dependent, so we assessed glucose sensitivity in a 5 -h glucose tolerance test (GTT) at 5-weeks post infection (pre-egg laying). We found that pre-patency, S. mansoni infection has no significant effect on glucose tolerance in normal chow fed mice as measured by area under the glucose excursion curve (AUC, Figure 6A). HFD increases blood glucose levels and decreases glucose tolerance (Figure 6A), while pre-patent S. mansoni infection has no significant effect on this increase. At the transition to chronic infection (10 weeks post infection), normal chow fed infected mice have lower fasting glucose levels and significantly improved whole-body glucose tolerance (Figure 6B). HFD significantly impairs glucose tolerance (as compared to chow uninfected controls), while $S$. mansoni infection of HFD fed mice restores glucose tolerance to a similar level as chow uninfected mice (Figure 6B). This is distinct from what has previously been reported, where $S$. mansoni infection of C57BL/6 mice improved glucose tolerance in HFD fed mice, but not normal low-fat chow fed mice. This difference suggests that there may be strain-specific differences in the control of glucose sensitivity. Since ApoE $\mathrm{E}^{-/-}$are prone to develop hyperlipidemia and atherosclerosis spontaneously as they age, it is possible that this propensity underlies the difference.

We then went back to the microarray pathway analysis and looked for pathway changes that could be expected to lead to improvement in glucose tolerance based on published reports. 
A
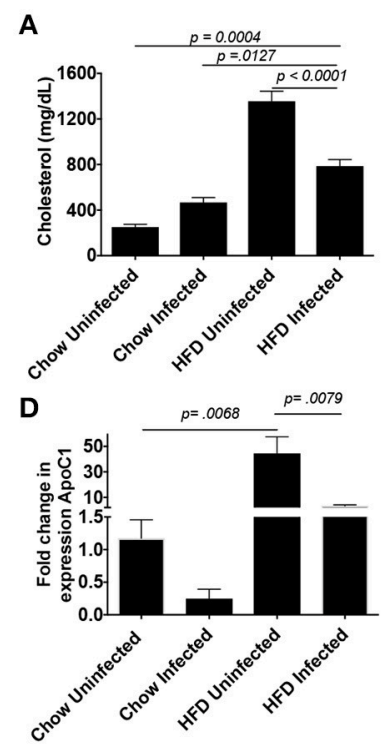

B

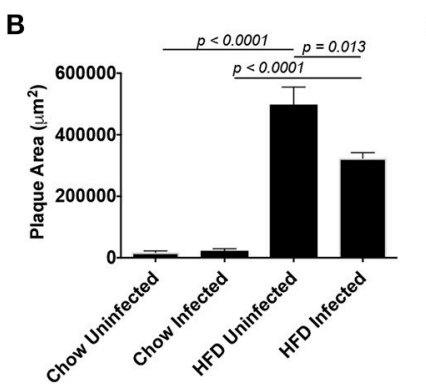

E

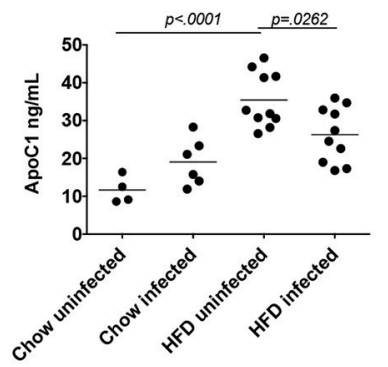

C

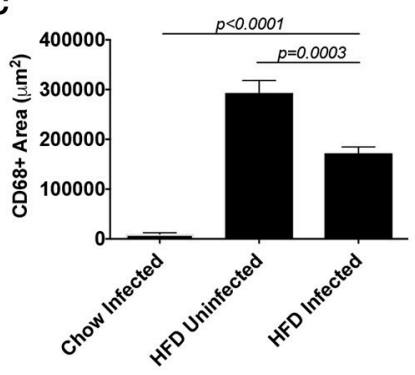

FIGURE 5 | S. mansoni infection reduces hyperlipidemia, plaque, and macrophage area and well as systemic ApoC1 production in HFD fed ApoE-/- mice. $\mathrm{ApoE}^{-/-}$male mice were fed HFD or standard chow diet for 10 days before infection with S. mansoni. At 10-weeks post infection, mice were sacrificed. (A) Fasting plasma cholesterol measurements. (B-C) Quantitation of plaque and macrophage area (CD68 ${ }^{+}$) from the aortic sinus. (D) QPCR analysis of ApoC1 in whole liver RNA, data are normalized to Chow infected animals. (E) Quantitation of ApoC1 in plasma at the time of sacrifice. All data shown are two combined experiments with 4-8 mice per group in each experiment. The experiment was performed 4 times. Statistical significance was calculated using ANOVA and post-tests.

We found that $S$. mansoni infection significantly alters the biosynthesis of amino acids in Kupffer cells (Figure 6C, pathway analysis Bonferroni corrected $p=0.006$ ), and one of the genes in that pathway, Psat1, stood out as a candidate for involvement in improving glucose tolerance as its transcript level is increased in infected $\mathrm{C} 57 \mathrm{BL} / 6$ mice $(\log \mathrm{FC}=5$,). The correlation between modulation of this transcript and infection was confirmed in our ApoE ${ }^{-/-}$model (Figure 6C). Hepatic psat1 transcript levels (measured in total liver RNA) have previously been shown to be reduced in both genetic and diet-induced models of diabetes, while global overexpression of PSAT1 improves insulin signaling and sensitivity (19). The glucagon signaling pathway is also significantly altered (Figure 6D, pathway Bonferroni corrected $p=0.015)$, in that pathway, $S$. mansoni infection decreases pygl expression while also increasing gys 1 transcripts. Genetic disruption of PYGL has previously been shown to improve glucose tolerance in vivo (32). While GYS1 is one of two enzymes responsible for glycogen synthesis, and small molecule induced activation of GYS1 in diabetic mice improves glucose tolerance (33). The combined effects of these two alterations would be predicted to lead to increased glucose tolerance and insulin sensitivity. We then asked whether gys 1 transcripts are similarly altered by $S$. mansoni infection of HFD fed $\mathrm{ApoE}^{-/-}$mice. $S$. mansoni infection leads to significantly higher gys 1 transcripts in the liver tissue of both chow and HFD fed mice (Figure 6E). Psat1 is similarly altered by infection (Figure 6F), with transcripts significantly higher in $S$. mansoni infected mice on both chow and HFD, although transcript levels of HFD infected mice are lower than those of chow infected. These data suggest that the $S$. mansoni induced transcriptional changes that were identified via the microarray also occur in the liver tissue of HFD fed $\mathrm{ApoE}^{-/-}$mice, supporting the hypothesis that $S$. mansoni induced alterations in hepatic macrophage metabolism are able to influence whole body metabolism and help protect from pathological changes generally associated with HFD induced metabolic disorders.

\section{DISCUSSION}

Overall, our data provide the first complete transcriptional analysis of Kupffer cells and perivascular macrophages in steady state and chronic S. mansoni infection. These data suggest that during S. mansoni infection, egg-induced M2 macrophages demonstrate profound alterations to their metabolic profile, with shifts in the balance of phospholipid and glucose metabolism as well as increased amino acid metabolism, and that these changes correlate with improvements in whole body cholesterol and glucose metabolism. While many of these alterations are shared between both perivascular macrophages and Kupffer cells, there are key genes that are differentially regulated in the two populations. Cbr2, Alox5, and F13a1 are all significantly up-regulated in Kupffer cells, but not in perivascular macrophages. Since Cbr2 and Alox are involved in arachidonic acid metabolism, it suggests that while both populations are alternatively activated, Kupffer cells specifically increase leukotriene production in the granuloma. Similarly, F13a1 has been previously implicated in wound healing (34), suggesting the Kupffer cells are more involved in the process of granuloma formation and resolution. Conversely, Lox is upregulated in both macrophage populations, but the increase 

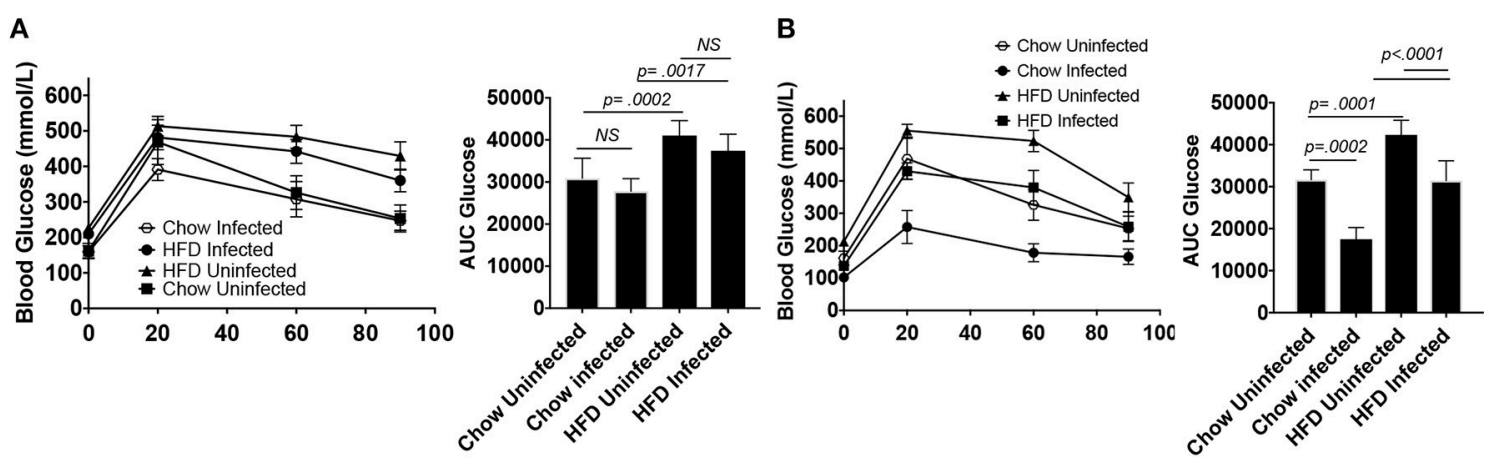

C Biosynthesis of Amino Acids

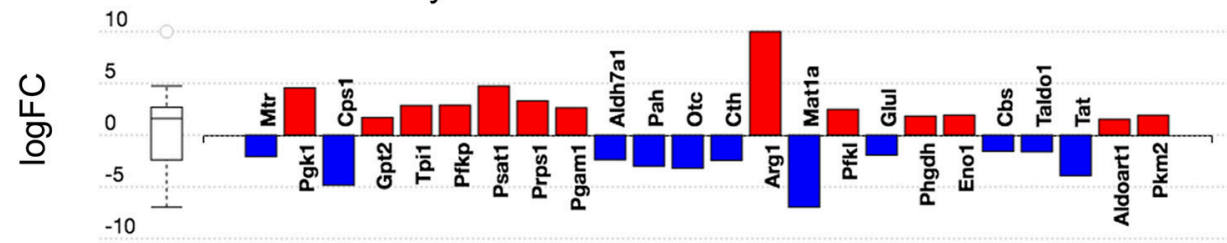

D

\section{Glucagon Signaling}

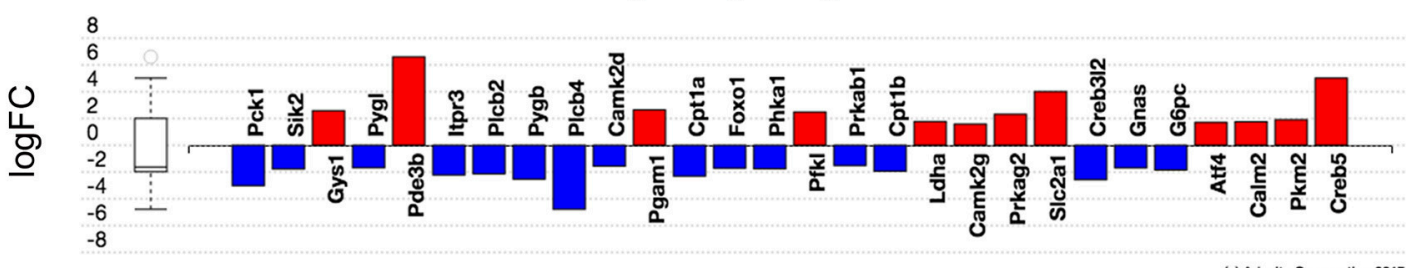

E
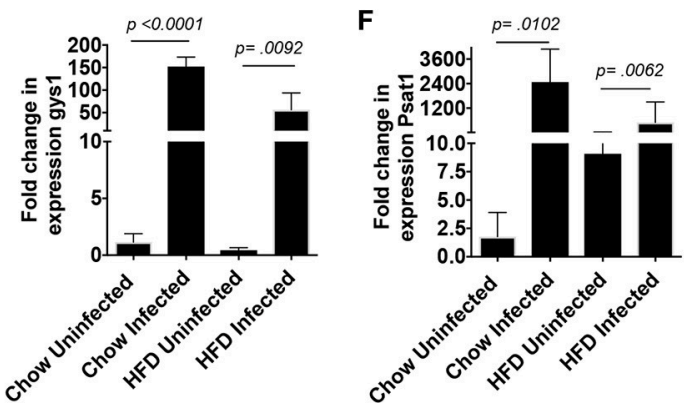

FIGURE 6 | S. mansoni infection improves glucose tolerance and alters hepatic metabolism toward glycogen storage and amino acid biosynthesis. ApoE ${ }^{-/-}$male mice were fed HFD or standard chow diet for 10 days before infection with S. mansoni. At 5 (A) and 10- (B) weeks post infection mice were fasted for $5 \mathrm{~h}$ and then administered an i.v GTT. (C,D) Perivascular macrophages and Kupffer cells were sorted from naïve and 10-week S. mansoni-infected C57BL/6 mice, RNA was extracted following Immgen protocols and populations were analyzed using whole-mouse genome Affymetrix Mouse Gene 2.0 ST Arrays. Significantly up (red) and down (blue) -regulated pathways were identified using Advaita Corporation iPathwayGuide. (E,F) QPCR analysis of gys1 and psat1 in whole liver RNA, data are normalized to Chow infected animals. Data in (A,B,E,F) are two combined experiments with 4-8 mice per group in each experiment and the experiment was performed 4 times. Statistical significance was calculated using ANOVA and post-tests.

in perivascular macrophages is far higher (8.3- vs. 2.1-fold), suggesting that perivascular macrophages are responding to greater oxidative stress during $S$. mansoni infection, a proposition that is supported by their tissue location. Many of the key markers of M2 activation (Chi313, Retnal, Mgl2) have a lower fold upregulation in perivascular macrophages, a finding that is matched by the frequency of these macrophages that are $\mathrm{CD}_{206}{ }^{+} \mathrm{CD}_{301}{ }^{+}$, suggesting that there may be degrees of alternative activation in the liver in the context of $S$. mansoni infection. The fact that only five genes (Subsets 1 and 2) are truly differentially regulated (opposite signs of significant fold changes) supports the hypothesis that after infection Kupffer cells and Perivascular macrophages act to a large degree in unison.

Recent work, using other models of M2 macrophage generation, have indicated that M2 macrophages rely on lipolysis 
driven fatty acid oxidation $(35,36)$ for the generation and maintenance of the M2 state. Our transcriptional analysis of Kupffer cells and perivascular macrophages suggest similar alterations are induced by chronic S. mansoni infection, but our data also provide the first evidence that alterations to hepatic macrophage metabolism either reflect or influence infectioninduced alterations to whole body metabolism. Macrophages are known to be critical sources of apolipoproteins like ApoC1 $(31,37)$, and LPS-induced inflammation has been linked with increased levels of plasma cholesterol and ApoC1 (37). Our data indicating that $S$. mansoni-induced M2 transcriptional changes reduce both ApoC1 production and plasma cholesterol is in concordance with the established paradigm of M1 and M2 polarization states as driving opposite pathological states, and with the hypothesis that M2 macrophages play a role in the resolution and modulation of atherosclerotic lesions (38-40).

The liver plays a critical role in whole body metabolism through its control of glycogen metabolism, with glycogen synthase (GYS1) and glycogen phosphorylase (PYGL) acting to respectively increase and decrease stored glycogen to maintain blood sugar homeostasis. Our pathway analysis revealed key alterations to both macrophage amino acid and glucagon signaling/metabolic pathways. We identified key genes (GYS1, PYGL, and PSAT1) from both pathways that have previously been demonstrated to play a role in glucose tolerance/ insulin sensitivity in models of obesity and diabetes $(19,41)$ and epidemiological studies (42-44). We hypothesize that the combined effects of $S$. mansoni-induced alterations to these three genes are at least partially responsible for infection-related improvements in glucose tolerance in both chow and HFD fed mice, a proposition that will be tested in future studies. These data raise the possibility that $S$. mansoni-induced polarization of liver macrophages can significantly alter whole body metabolism. While multiple previous studies in humans $(26,45,46)$ and mice $(15,16,27)$ have indicated that helminth infections in general, and schistosomes in specific, modulate susceptibility to

\section{REFERENCES}

1. Sica A, Mantovani A. Macrophage plasticity and polarization: in vivo veritas. J Clin Invest. (2012) 122:787-95. doi: 10.1172/JCI59643

2. Gordon S. Alternative activation of macrophages. Nat Rev Immunol. (2003) 3:23-35. doi: 10.1038/nri978

3. Gordon S, Martinez FO. Alternative activation of macrophages: mechanism and functions. Immunity (2010) 32:593-604. doi: 10.1016/j.immuni.2010.05.007

4. Gautier EL, Shay T, Miller J, Greter M, Jakubzick C, Ivanov S, et al. Geneexpression profiles and transcriptional regulatory pathways that underlie the identity and diversity of mouse tissue macrophages. Nat Immunol. (2012) 13:1118-28. doi: 10.1038/ni.2419

5. Leopold Wager CM, Wormley FLJr. Classical versus alternative macrophage activation: the Ying and the Yang in host defense against pulmonary fungal infections. Mucosal Immunol. (2014) 7:1023-35. doi: 10.1038/mi.2014.65

6. Martinez FO, Gordon S. The M1 and M2 paradigm of macrophage activation: time for reassessment. F1000Prime Rep (2014) 6:13. doi: 10.12703/P6-13

7. Murray PJ, Wynn TA. Protective and pathogenic functions of macrophage subsets. Nat Rev Immunol. (2011) 11:723-37. doi: 10.1038/nri3073 the development of metabolic diseases, a molecular mechanism underlying this modulation has not been identified. We postulate that S. mansoni-induced M2 polarization of hepatic macrophages are involved in protection from HFD induced hyperlipidemia, atherosclerosis, and glucose intolerance. Future studies will focus on thoroughly understanding the infectioninduced transcriptional changes to other tissue macrophage populations and the broader monocyte lineage.

\section{AUTHOR CONTRIBUTIONS}

KF, EP, and GR conceived of the project. KF, AR, DC-S, and GR planned the experiments. DC-S, AE, AR, and KF performed the experiments. KF, BR, DC-S, and AE analyzed the data. DC-S, KF, and $\mathrm{BR}$ wrote the manuscript.

\section{FUNDING}

The work was supported by Purdue University, and a Scientist Development Grant from the American Heart Association to KF (14SDG18230012), R37 AI049653 and R01 HL118206 to GR, and AI32573 to EP.

\section{ACKNOWLEDGMENTS}

We thank Eyal Amiel and Bart Everts for helpful discussions. B. glabrata snails were provided by the NIAID Schistosomiasis Resource Center of the Biomedical Research Institute (Rockville, MD) through NIH-NIAID Contract HHSN272201000005I for distribution through BEI Resources.

\section{SUPPLEMENTARY MATERIAL}

The Supplementary Material for this article can be found online at: https://www.frontiersin.org/articles/10.3389/fimmu. 2018.02580/full\#supplementary-material
8. Gause WC, Wynn TA, Allen JE. Type 2 immunity and wound healing: evolutionary refinement of adaptive immunity by helminths. Nat Rev Immunol. (2013) 13:607-14. doi: 10.1038/nri3476

9. Brunet LR, Finkelman FD, Cheever AW, Kopf MA, Pearce EJ. IL-4 protects against TNF-alpha-mediated cachexia and death during acute schistosomiasis. J Immunol. (1997) 159:777-85.

10. Herbert DR, Holscher C, Mohrs M, Arendse B, Schwegmann A, Radwanska $M$, et al. Alternative macrophage activation is essential for survival during schistosomiasis and downmodulates $\mathrm{T}$ helper 1 responses and immunopathology. Immunity (2004) 20:623-35. doi: 10.1016/S1074-7613(04)00107-4

11. Pesce JT, Ramalingam TR, Mentink-Kane MM, Wilson MS, El Kasmi KC, Smith AM, et al. Arginase-1-expressing macrophages suppress Th2 cytokine-driven inflammation and fibrosis. PLoS Pathog (2009) 5:e1000371. doi: 10.1371/journal.ppat.1000371

12. Fairfax KC, Amiel E, King IL, Freitas TC, Mohrs M, Pearce EJ. IL10R blockade during chronic Schistosomiasis mansoni results in the loss of B cells from the liver and the development of severe pulmonary disease. PLoS Pathog (2012) 8:e1002490. doi: 10.1371/journal.ppat. 1002490 
13. Wynn TA, Thompson RW, Cheever AW, Mentink-Kane MM. Immunopathogenesis of schistosomiasis. Immunol Rev. (2004) 201:156-67. doi: 10.1111/j.0105-2896.2004.00176.x

14. Martins da Fonseca CS, Pimenta Filho AA, dos Santos BS, da Silva CA, Domingues AL, Owen JS, et al. Human plasma lipid modulation in Schistosomiasis mansoni depends on apolipoprotein E polymorphism. PLoS ONE (2014) 9:e101964. doi: 10.1371/journal.pone.0101964

15. Stanley RG, Jackson CL, Griffiths K, Doenhoff MJ. Effects of Schistosoma mansoni worms and eggs on circulating cholesterol and liver lipids in mice. Atherosclerosis (2009) 207:131-8. doi: 10.1016/j.atherosclerosis.2009.04.037

16. Doenhoff MJ, Stanley RG, Griffiths K, Jackson CL. An anti-atherogenic effect of Schistosoma mansoni infections in mice associated with a parasite-induced lowering of blood total cholesterol. Parasitology (2002) 125(Pt 5):415-21. doi: $10.1017 /$ S0031182002002275

17. Potteaux S, Gautier EL, Hutchison SB, van Rooijen N, Rader DJ, Thomas MJ, et al. Suppressed monocyte recruitment drives macrophage removal from atherosclerotic plaques of Apoe ${ }^{-/-}$mice during disease regression. J Clin Invest. (2011) 121:2025-36. doi: 10.1172/JCI43802

18. Fairfax KC, Everts B, Smith AM, Pearce EJ. Regulation of the development of the Hepatic B Cell compartment during Schistosoma mansoni infection. J Immunol (2013). doi: 10.4049/jimmunol.1301357

19. Yu J, Xiao F, Guo Y, Deng J, Liu B, Zhang Q, et al. Hepatic phosphoserine aminotransferase 1 regulates insulin sensitivity in mice via tribbles homolog 3. Diabetes (2015) 64:1591-602. doi: $10.2337 / \mathrm{db} 14-1368$

20. Heng TS, Painter M, Immunological Genome Project CW. The Immunological Genome Project: networks of gene expression in immune cells. Nat Immunol. (2008) 9:1091-4. doi: 10.1038/ni1008-1091

21. Girgis NM, Gundra UM, Ward LN, Cabrera M, Frevert U, Loke P. Ly6C(high) monocytes become alternatively activated macrophages in schistosome granulomas with help from $\mathrm{CD}^{4+}$ cells. PLoS Pathog (2014) 10:e1004080. doi: 10.1371/journal.ppat.1004080

22. Nascimento M, Huang SC, Smith A, Everts B, Lam W, Bassity E, et al. Ly6Chi monocyte recruitment is responsible for Th2 associated host-protective macrophage accumulation in liver inflammation due to schistosomiasis. PLoS Pathog (2014) 10:e1004282. doi: 10.1371/journal.ppat. 1004282

23. Wang J, Kubes P. A reservoir of mature cavity macrophages that can rapidly invade visceral organs to affect tissue repair. Cell (2016) 165:668-78. doi: 10.1016/j.cell.2016.03.009

24. Langhans W. Role of the liver in the control of glucose-lipid utilization and body weight. Curr Opin Clin Nutr Metab Care (2003) 6:449-55. doi: 10.1097/01.mco.0000078993.96795.16

25. Postic C, Dentin R, Girard J. Role of the liver in the control of carbohydrate and lipid homeostasis. Diabetes Metab. (2004) 30:398-408. doi: 10.1016/S1262-3636(07)70133-7

26. Chen Y, Lu J, Huang Y, Wang T, Xu Y, Xu M, et al. Association of previous schistosome infection with diabetes and metabolic syndrome: a crosssectional study in rural China. J Clin Endocrinol Metab. (2013) 98:E283-287. doi: 10.1210/jc.2012-2517

27. Hussaarts L, Garcia-Tardon N, van Beek L, Heemskerk MM, Haeberlein S, van der Zon GC, et al. Chronic helminth infection and helminthderived egg antigens promote adipose tissue M2 macrophages and improve insulin sensitivity in obese mice. FASEB J. (2015) 29:3027-39. doi: 10.1096/fj.14-266239

28. Nakashima Y, Plump AS, Raines EW, Breslow JL, Ross R. ApoE-deficient mice develop lesions of all phases of atherosclerosis throughout the arterial tree. Arterioscler Thromb. (1994) 14:133-40. doi: 10.1161/01.ATV.14.1.133

29. Swirski FK, Pittet MJ, Kircher MF, Aikawa E, Jaffer FA, Libby P, et al. Monocyte accumulation in mouse atherogenesis is progressive and proportional to extent of disease. Proc Natl Acad Sci USA. (2006) 103:10340-5. doi: 10.1073/pnas.0604260103

30. Tacke F, Alvarez D, Kaplan TJ, Jakubzick C, Spanbroek R, Llodra J, et al. Monocyte subsets differentially employ CCR2, CCR5, and CX3CR1 to accumulate within atherosclerotic plaques. J Clin Invest. (2007) 117:185-94. doi: $10.1172 /$ JCI28549

31. Westerterp M, de Haan W, Berbee JF, Havekes LM, Rensen PC. Endogenous apoC-I increases hyperlipidemia in apoE-knockout mice by stimulating VLDL production and inhibiting LPL. J Lipid Res. (2006) 47:1203-11. doi: 10.1194/jlr.M500434-JLR200
32. Kelsall IR, Rosenzweig D, Cohen PT. Disruption of the allosteric phosphorylase a regulation of the hepatic glycogen-targeted protein phosphatase 1 improves glucose tolerance in vivo. Cell Signal. (2009) 21:1123-34. doi: 10.1016/j.cellsig.2009.03.001

33. Nakano K, Takeshita S, Kawasaki N, Miyanaga W, Okamatsu Y, Dohi M, et al. AJS1669, a novel small-molecule muscle glycogen synthase activator, improves glucose metabolism and reduces body fat mass in mice. Int $\mathrm{J} \mathrm{Mol}$ Med. (2017) 39:841-50. doi: 10.3892/ijmm.2017.2909

34. Andersson C, Kvist PH, McElhinney K, Baylis R, Gram LK, Pelzer $\mathrm{H}$, et al. Factor XIII transglutaminase supports the resolution of mucosal damage in experimental colitis. PLoS ONE (2015) 10:e0128113. doi: 10.1371/journal.pone.0128113

35. Vats D, Mukundan L, Odegaard JI, Zhang L, Smith KL, Morel CR, et al. Oxidative metabolism and PGC- $1 \mathrm{~b} \beta$ attenuate macrophage-mediated inflammation. Cell Metab. (2006) 4:13-24. doi: 10.1016/j.cmet.2006.05.011

36. Huang SC, Everts B, Ivanova Y, O'Sullivan D, Nascimento M, Smith AM, et al. Cell-intrinsic lysosomal lipolysis is essential for alternative activation of macrophages. Nat Immunol. (2014) 15:846-55. doi: 10.1038/ni.2956

37. Westerterp M, Berbee JF, Pires NM, van Mierlo GJ, Kleemann R, Romijn JA, et al. Apolipoprotein C-I is crucially involved in lipopolysaccharide-induced atherosclerosis development in apolipoprotein E-knockout mice. Circulation (2007) 116:2173-81. doi: 10.1161/CIRCULATIONAHA.107.693382

38. Chinetti-Gbaguidi G, Baron M, Bouhlel MA, Vanhoutte J, Copin C, Sebti $\mathrm{Y}$, et al. Human atherosclerotic plaque alternative macrophages display low cholesterol handling but high phagocytosis because of distinct activities of the PPARgamma and LXRalpha pathways. Circ Res. (2011) 108:985-95. doi: 10.1161/CIRCRESAHA.110.233775

39. Chinetti-Gbaguidi G, Colin S, Staels B. Macrophage subsets in atherosclerosis. Nat Rev Cardiol. (2015) 12:10-7. doi: 10.1038/nrcardio.2014.173

40. Roma-Lavisse C, Tagzirt M, Zawadzki C, Lorenzi R, Vincentelli A, Haulon S, et al. M1 and M2 macrophage proteolytic and angiogenic profile analysis in atherosclerotic patients reveals a distinctive profile in type 2 diabetes. Diab Vasc Dis Res. (2015) 12:279-89. doi: 10.1177/1479164115582351

41. Gasa R, Clark C, Yang R, DePaoli-Roach AA, Newgard CB. Reversal of dietinduced glucose intolerance by hepatic expression of a variant glycogentargeting subunit of protein phosphatase-1. J Biol Chem. (2002) 277:1524-30. doi: 10.1074/jbc.M107744200

42. Schalin-Jantti C, Harkonen M, Groop LC. Impaired activation of glycogen synthase in people at increased risk for developing NIDDM. Diabetes (1992) 41:598-604. doi: 10.2337/diab.41.5.598

43. Shimomura H, Sanke T, Ueda K, Hanabusa T, Sakagashira S, Nanjo K. A missense mutation of the muscle glycogen synthase gene (M416V) is associated with insulin resistance in the Japanese population. Diabetologia (1997) 40:947-52. doi: 10.1007/s001250050772

44. St-Onge J, Joanisse DR, Simoneau JA. The stimulation-induced increase in skeletal muscle glycogen synthase content is impaired in carriers of the glycogen synthase XbaI gene polymorphism. Diabetes (2001) 50:195-8. doi: 10.2337/diabetes.50.1.195

45. Aravindhan V, Mohan V, Surendar J, Muralidhara Rao M, Pavankumar $\mathrm{N}$, Deepa $\mathrm{M}$, et al. Decreased prevalence of lymphatic filariasis among diabetic subjects associated with a diminished pro-inflammatory cytokine response (CURES 83). PLoS Negl Trop Dis. (2010) 4:e707. doi: 10.1371/journal.pntd.0000707

46. Wiria AE, Hamid F, Wammes LJ, Prasetyani MA, Dekkers OM, May L, et al. (2015). Infection with soil-transmitted helminths is associated with increased insulin sensitivity. PLOS ONE 10:e0127746. doi: 10.1371/journal.pone.0127746

Conflict of Interest Statement: The authors declare that the research was conducted in the absence of any commercial or financial relationships that could be construed as a potential conflict of interest.

Copyright (c) 2018 Cortes-Selva, Elvington, Ready, Rajwa, Pearce, Randolph and Fairfax. This is an open-access article distributed under the terms of the Creative Commons Attribution License (CC BY). The use, distribution or reproduction in other forums is permitted, provided the original author(s) and the copyright owner(s) are credited and that the original publication in this journal is cited, in accordance with accepted academic practice. No use, distribution or reproduction is permitted which does not comply with these terms. 